www.nature.com/bmt

\title{
Erratum
}

\section{Immunomodulatory effects of human foetal liver-derived mesenchymal stem cells}

\author{
C Götherström, O Ringdén, M Westgren, C Tammik and K Le Blanc
}

Bone Marrow Transplantation (2004) 33, 1167. doi:10.1038/sj.bmt.1704318

Correction to: Bone Marrow Transplantation (2003) 32, 265-272. doi: 10.1038/sj.bmt.1704111
Unfortunately due to an error, Figure 2 was published incorrectly.

The correct version is reprinted below:

The publisher would like to apologise for any inconvenience caused.
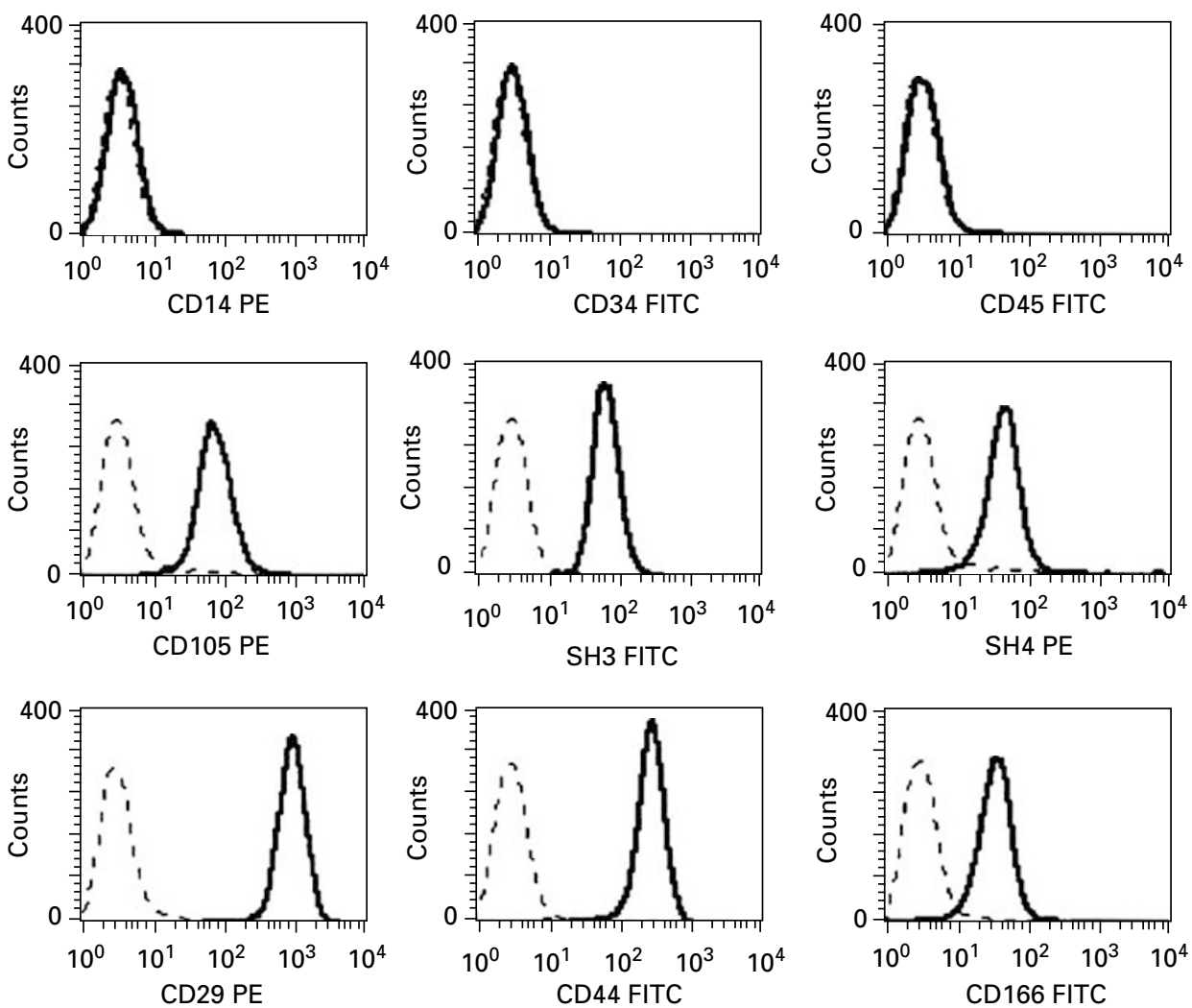

Figure 2 Immunophenotype of foetal MSC by flow cytometry analysis. Cultured MSC from six human foetal livers between passage two and nine were stained with monoclonal antibodies against CD29, CD44, CD166, CD105 (also called SH-2), SH-3, SH-4, CD14, CD34 and CD45. Dotted lines indicate isotype-matched mouse IgG antibody control staining. This is a typical experiment with foetal MSC. Human foetal MSC were positive for CD29, CD44, CD166, CD105, SH-3 and SH-4 and negative for CD14, CD34 and CD45. 